'Departamento de Nutrición, Diabetes y Metabolismo. Pontificia Universidad Católica de Chile. Santiago, Chile. ${ }^{2}$ Servicio de Medicina. Hospital San Juan de Dios de Los Andes. Los Andes, Chile. ${ }^{3}$ Centro de Nutrición y Diabetes. Clínica Alemana de Santiago. Santiago, Chile. anterno de Medicina. Escuela de Medicina. Pontificia Universidad Católica de Chile. Santiago, Chile.

Trabajo no recibió financiamiento. Los autores declaran no tener conflictos de interés.

Recibido el 2 de octubre de 2020, aceptado el 6 de enero de 2021 .

Correspondencia a: Alberto Maiz Gurruchaga. Diagonal Paraguay 362, $4^{\circ}$ Piso.

Departamento de Nutrición, Diabetes y Metabolismo. Pontificia Universidad Católica de Chile. Santiago, Chile. maiz@med.puc.cl

\section{Prevalencia de hiperglicemia y su relación con el tiempo de estadía en un hospital terciario}

\author{
BRUNO GRASSI ${ }^{1}$, PAOLA ÁLVAREZ ${ }^{2}$, FERNANDA KARAª, \\ KRISTEL STRODTHOFF ${ }^{3}$, ALBERTO MAIZ ${ }^{1}$
}

\begin{abstract}
Background: Hyperglycemia during hospital stay is associated with adverse outcomes. Aim: To characterize the frequency of hyperglycemia in a tertiary hospital and to correlate it with length of hospital stay (LOS). Material and Methods: Review of medical records of hospitalized patients. Demographic data and laboratory data, previous diabetes mellitus (DM) history, current main diagnosis, unit of hospitalization and the two highest capillary blood glucose values from the analyzed period were recorded for each patient. LOS was obtained from electronic clinical records. Results: 210 subjects, aged $60 \pm 19$ years (104 women) were included. 113 patients (54\%) developed hyperglycemia $\geq 140$ $\mathrm{mg} / \mathrm{L}$. Thirty one percent of these had a previous history of diabetes and 29\% had stress hyperglycemia (SHG). Patients with a history of DM had a higher average blood glucose than those with SHG (238.9 and $178.2 \mathrm{mg} / \mathrm{dL}$, respectively, $p<0.01$ ) and a greater percentage of cases with a blood glucose above 180 $m g / d L$ (72 and $40.0 \%$, respectively, $p<0.01$ ). Hospital LOS was significantly longer in patients with hyperglycemia $\geq 140 \mathrm{mg} / \mathrm{dL}$ as compared with those with normoglycemia (29.3 and 12.8 days, respectively, $p<0.01$ ). This association remained significant when introduced in a linear regression analysis including diagnosis, decreased glomerular filtration rate (GFR) and hospitalization unit ( $p<0.01)$. Conclusions: Hyperglycemia during hospitalization affects more than half of hospitalized patients and is associated with a longer length of stay.

(Rev Med Chile 2021; 149: 210-216)
\end{abstract}

Key words: Hospitalization; Hyperglycemia; Inpatients; Registries.
L a hiperglicemia es un problema de altísima prevalencia en población hospitalaria y se ha asociado a desenlaces adversos ${ }^{1}$. Esta situación puede deberse a la alta tasa de obesidad, prediabetes y diabetes mellitus (DM) de nuestra población ${ }^{2}$. Sin embargo, aproximadamente la mitad de los casos de hiperglicemia en el hospital ocurre en pacientes sin antecedentes de DM, y se explicarían por las hormonas contrarreguladoras secretadas en relación al estrés derivado de enfermedades causales de la hospitalización, las que, además, pueden tener terapias complejas que generen efectos metabólicos no deseados ${ }^{1,3}$.

Concentraciones elevadas de glucosa en sangre han mostrado disminuir la función de neutrófilos, particularmente su capacidad fagocítica ${ }^{4}$. La hiperglicemia generaría, además, un aumento en la producción de radicales libres en el endotelio, amplificando el daño en los tejidos 5 . Ambos hechos parecieran explicar los fenómenos observados en relación a la hiperglicemia.

Desde una perspectiva clínica, el nivel de gli- 
cemia de ingreso al hospital y durante la estadía ha sido asociado a hospitalizaciones significativamente más prolongadas ${ }^{3,6}$. También, se ha descrito una relación lineal y proporcional con la mortalidad durante la estadía, tanto en pacientes con DM previamente diagnosticada como en pacientes con hiperglicemia sin antecedentes de $\mathrm{DM}^{3,7}$. Se ha reportado mayor mortalidad en relación a hiperglicemia incluso un año después de la hospitalización ${ }^{8}$. Todos estos desenlaces adversos se deberían principalmente a complicaciones infecciosas s.6,7,8. $^{3,7}$.

A principios de la década antepasada, se evaluó el significado de tratar pacientes críticos quirúrgicos y médicos con protocolos de manejo intensivo de las hiperglicemias con insulina endovenosa. La evolución y complicaciones de aquellos que lograron glicemias promedio menores a $110 \mathrm{mg} /$ dL se compararon a pacientes con un tratamiento convencional de la hiperglicemia, la que se mantuvo en promedio sobre $180 \mathrm{mg} / \mathrm{dL}$. El grupo con tratamiento intensivo mostró una significativa reducción en los tiempos de estadía en cuidados intensivos, en tiempos de estadía hospitalaria y en la mortalidad ${ }^{9,10}$. Algunos años más tarde, los investigadores del estudio NICE-SUGAR exploraron el nivel recomendable y seguro que se debería tener para el control glicémico en pacientes críticos de altísima gravedad. Se encontró que aquellos que mantuvieron glicemias entre 140 y $180 \mathrm{mg} / \mathrm{dL}$ tuvieron una sobrevida mayor que la del grupo tratado para alcanzar una meta de 80 a $110 \mathrm{mg} / \mathrm{dL}$. Esto fue atribuido a un mayor número de hipoglicemias y eventos cardiovasculares asociados a los niveles más bajos de glicemias ${ }^{11}$. La presencia de hipoglicemia durante la hospitalización también se ha asociado a mayores estadías hospitalarias y a mayor mortalidad, tanto intrahospitalaria como posterior al alta ${ }^{12}$.

En consecuencia con lo expuesto, guías clínicas internacionales definen una glicemia mayor a $140 \mathrm{mg} / \mathrm{dL}$ como "hiperglicemia" en contexto hospitalario, recomendando una meta deseada para el control glicémico de 140 a $180 \mathrm{mg} / \mathrm{dL}$ y con indicación de tratamiento con insulina en todo paciente con hiperglicemia $>180 \mathrm{mg} / \mathrm{dL}$. Las mismas guías recomiendan que a todo paciente hospitalizado con hiperglicemia se le mida una hemoglobina glicosilada para evaluar la presencia previa de $\mathrm{DM}^{1}$.

El presente estudio tuvo como objetivo carac- terizar en forma transversal el nivel de glicemia que presentan los pacientes ingresados en todas las camas de los distintos servicios de un hospital terciario. Se detallaron variables asociadas a la presencia de hiperglicemia, ya sea de la patología de ingreso del paciente, o del servicio en el que se encuentran hospitalizados y su relación con el tiempo de estadía hospitalaria.

\section{Material y Método}

El presente estudio es descriptivo de tipo transversal. Por su carácter observacional, fue presentado y aprobado para dispensa por el Comité Ético Científico de Ciencias de la Salud de la Pontificia Universidad Católica de Chile. No se obtuvieron datos sensibles, y la información fue anonimizada en una base de datos con números, con acceso solo para los investigadores.

El equipo investigador identificó en los registros de admisión a todos los pacientes adultos ingresados en el Hospital Clínico de la Pontificia Universidad Católica de Chile entre el 2 y 6 de diciembre de 2013. Se incluyeron aquellos hospitalizados en servicios médico-quirúrgicos de baja y mediana complejidad y en servicios de paciente crítico. Se excluyeron aquellos hospitalizados en pediatría, obstetricia, unidades de permanencia transitoria y aquellos en que su ficha no contara con registros glicémicos.

Las fichas médicas y de enfermería de cada paciente fueron revisadas una única vez durante el período descrito con una planilla estandarizada. Se registraron sexo, edad, índice de masa corporal (IMC), servicio de hospitalización, antecedente de DM, la patología de ingreso, creatinina plasmática, hemoglobina glicosilada (HbAlc) de la hospitalización o hasta dos semanas previas y los dos valores más altos de glicemia capilar. El estado nutricional de los pacientes se categorizó en normopeso, bajo peso o enflaquecido y sobrepeso-obesidad, considerando IMC y edad de los pacientes, según consenso vigente ${ }^{13}$. La tasa de filtración glomerular (TFG) se calculó con la fórmula $\mathrm{CKD}-\mathrm{EPI}^{14}$. Se registró la vía preponderante de alimentación del paciente, clasificada en régimen cero, vía oral, nutrición enteral o nutrición parenteral. El esquema de tratamiento de la hiperglicemia se registró como: sin tratamiento, con insulina regular o rápida cada $6 \mathrm{~h}$, insulina en 
esquema basal bolo, bomba de infusión continua de insulina endovenosa y uso de hipoglicemiantes orales. El tiempo de estadía hospitalaria se obtuvo a posteriori de registros electrónicos, usando las fechas de ingreso y de egreso correspondientes a la hospitalización.

Se calculó el promedio de las dos mayores glicemias capilares registradas para cada paciente. Estos valores se clasificaron según el consenso para pacientes hospitalizados en: normoglicémicos con glicemia capilar $\leq 140 \mathrm{mg} / \mathrm{dL}$, como hiperglicemia para los valores mayores o iguales a esta cifra y estos, a su vez, se estratificaron en rangos entre 141 y $180 \mathrm{mg} / \mathrm{dL}$, entre 181 y $250 \mathrm{mg} / \mathrm{dL}$ y mayor a 250 $\mathrm{mg} / \mathrm{dL}$. Se consideró como "DM previa" cuando existía este antecedente o cuando la $\mathrm{HbAlc}$ fuera mayor a $6,5 \%{ }^{1}$.

Se compararon los promedios glicémicos $y$ nivel de gravedad de la hiperglicemia entre pacientes con hiperglicemia de estrés (HGE) o DM previa, con la patología de ingreso, definida como infecciosa, oncohematológica, cardiovascular, neurológica no vascular, metabólico-renal, quirúrgica u otros. También, con la unidad de ingreso de la hospitalización del paciente, definida como crítico (incluyendo intermedios, intensivos y unidad coronaria) o sala de cuidados generales médico-quirúrgicos y, finalmente, con el tiempo de estadía hospitalaria.

\section{Análisis estadístico}

Las variables continuas se expresan como medias con sus respectivas desviaciones estándar y se calculan diferencias de medias (MD). Las variables dicotómicas se expresan como proporciones. Se utilizó t-test para dos muestras independientes con variables continuas, test ANOVA para comparaciones múltiples, y test exacto de Fisher para evaluar riesgos relativos con variables dicotómicas. Para analizar el tiempo de estadía hospitalaria y otras variables que podrían explicarlo se utilizó un modelo de regresión lineal. Los análisis estadísticos se realizaron utilizando el software IBM SPSS Statistics para Mac, version 25 (IBM Corp., Armonk, NY, USA).

\section{Resultados}

El Hospital Clínico de la Pontificia Universidad Católica de Chile contaba con 380 camas en el pe- ríodo del estudio, de las cuales, 274 cumplían con criterios de inclusión. La muestra final fue de 210 pacientes, ya que en 64 camas no había paciente o este no tenía registro de glicemias.

Las características generales de la muestra: sexo, edad, IMC, antecedentes de $\mathrm{DM}$, presencia de TFG $<60 \mathrm{mg} / \mathrm{dL}$, diagnóstico de ingreso, servicios de hospitalización y la vía de alimentación, se muestran en la Tabla 1.

$\mathrm{Al}$ analizar el nivel de las glicemias de todos los pacientes hospitalizados, $97(46,2 \%)$ se encontraban con normoglicemia $\leq 140 \mathrm{mg} / \mathrm{dL}$. El resto $(113$ pacientes, 53,8\%) tenía hiperglicemia $>140 \mathrm{mg} /$ $\mathrm{dL}$, que se distribuyeron en: 51 sujetos $(24,2 \%)$

Tabla 1. Características demográficas de la muestra de pacientes y datos clínicos sobre la hospitalización

\begin{tabular}{|c|c|c|}
\hline Variable & & Valor \\
\hline Edad (años) & & $60 \pm 19$ \\
\hline $\begin{array}{l}\text { Sexo } \\
\text { Femenino } \\
\text { Masculino }\end{array}$ & n (\%) & $\begin{array}{l}104(49,6) \\
106(50,4)\end{array}$ \\
\hline IMC $\left(\mathrm{kg} / \mathrm{m}^{2}\right)$ & & $26,6 \pm 5,5$ \\
\hline $\begin{array}{l}\text { Categorización estado nutricional } \\
\text { Normopeso } \\
\text { Sobrepeso u obesidad } \\
\text { Enflaquecido }\end{array}$ & n (\%) & $\begin{array}{l}85(40,5) \\
95(45,2) \\
30(14,3)\end{array}$ \\
\hline $\begin{array}{l}\text { Diagnóstico previo de DM o } \\
\mathrm{HbA} 1 \mathrm{c}>6,5 \%\end{array}$ & n (\%) & $67(31,9)$ \\
\hline $\begin{array}{l}\text { Insuficiencia renal* } \\
\text { (TFG }<60 \mathrm{~mL} / \mathrm{min})\end{array}$ & n (\%) & $58(27,6)$ \\
\hline $\begin{array}{l}\text { Diagnóstico de ingreso } \\
\text { Infecciosa } \\
\text { Onco-hematológico } \\
\text { Cardiovascular } \\
\text { Neurológico no vascular } \\
\text { Metabólico-renal } \\
\text { Quirúrgica } \\
\text { Otros }\end{array}$ & n (\%) & $\begin{array}{rr}50 & (23,8) \\
37 & (17,6) \\
44 & (21,0) \\
26 & (12,4) \\
12 \quad(5,7) \\
32 & (15,2) \\
9 & (4,3)\end{array}$ \\
\hline $\begin{array}{l}\text { Vía de alimentación } \\
\text { Regimen cero } \\
\text { Vía oral exclusiva } \\
\text { Nutrición enteral } \\
\text { Nutrición parenteral total }\end{array}$ & $\mathrm{n}\left({ }^{\circ}\right.$ & $\begin{array}{r}24(11,4) \\
159(75,7) \\
17(8,1) \\
10 \quad(4,8)\end{array}$ \\
\hline $\begin{array}{l}\text { Unidad de hospitalización } \\
\text { Sala médico-quirúrgico } \\
\text { Crítico (intermedio, coronaria o UC }\end{array}$ & $\begin{array}{l}\mathrm{n}(\%) \\
\mathrm{JCl})\end{array}$ & $\begin{array}{r}154(73,3) \\
56(26,7)\end{array}$ \\
\hline
\end{tabular}

*En 28 pacientes no se contaba con información de creatinina durante la hospitalización. 
con hiperglicemia leve (entre 141-180 mg/dL), 40 casos $(19,1 \%)$ con hiperglicemia moderada (entre 180 y $250 \mathrm{mg} / \mathrm{dL})$ y $22(10,5 \%)$ con hiperglicemia grave $(>250 \mathrm{mg} / \mathrm{dL})$. Esta distribución de presenta en la Figura 1.

Cabe destacar que de los 62 pacientes con hiperglicemia moderada y grave $(>180 \mathrm{mg} / \mathrm{dL})$, que según las normas de consenso citadas debieron

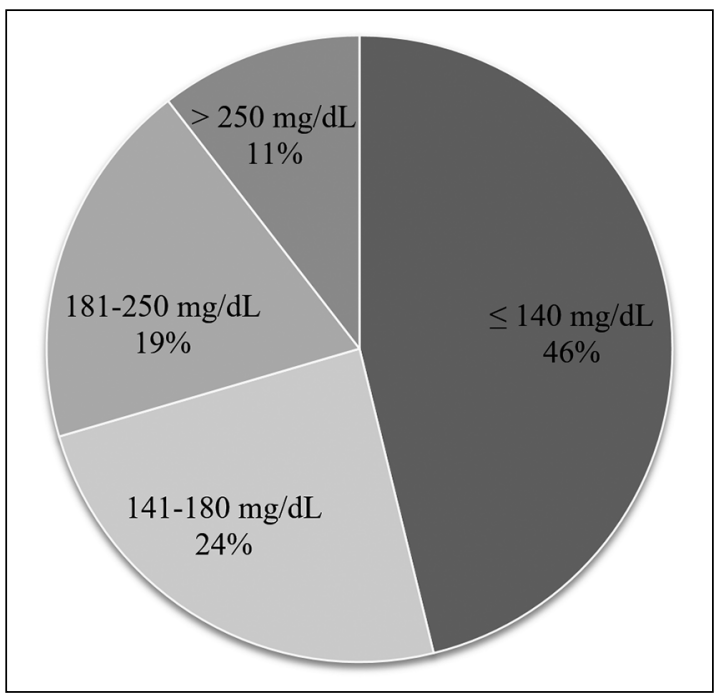

Figura 1. Rangos de glicemias en 210 pacientes adultos hospitalizados. Se muestra la distribución de 210 pacientes según rangos de glicemia. Se observa que el $54 \%$ de los pacientes presentan cifras de hiperglicemia $\geq 140 \mathrm{mg} / \mathrm{dL}$, y que $30 \%$ del total se encuentran en valores que requieren ser tratados con insulina (glicemia $>180 \mathrm{mg} / \mathrm{dL}$ ).

Tabla 2. Tipos de terapia hipoglicemiante en 113 pacientes con hiperglicemia mayor a $140 \mathrm{mg} / \mathrm{dL}$ y esquemas de insulinoterapia en 48 pacientes tratados con insulina

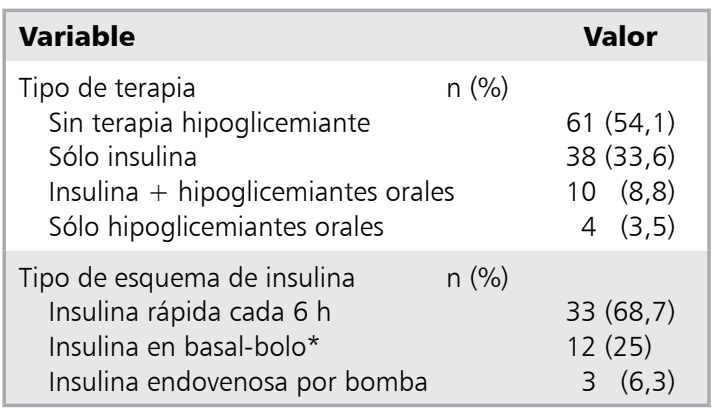

*Incluye pacientes con esquema basal-nutricional-correccional y basal-correccional. estar con terapia insulínica para control glicémico, solo 41 casos $(66,1 \%)$ estaban recibiendo algún esquema de insulina. En relación a pacientes con hiperglicemia leve, el $15,7 \%$ de ellos estaba recibiendo terapia con insulina. En la Tabla 2, se muestran los esquemas de tratamiento de los pacientes que presentaban hiperglicemia $>140 \mathrm{mg} /$ $\mathrm{dL}$, categorizados como: sin terapia, con insulina, con insulina + hipoglicemiantes orales o solo con hipoglicemiantes orales. Posteriormente, en la misma tabla se muestran los distintos esquemas de insulina utilizados.

De los 113 pacientes $(53,8 \%)$ con hiperglicemia $>140 \mathrm{mg} / \mathrm{dL}$, solo 53 tenían diagnóstico previo de DM y la diferencia (60 pacientes; $28,6 \%$ del total analizado) fueron diagnosticados como pacientes con hiperglicemia del estrés (HGE). Cabe destacar que en la muestra se registraron, además, 14 pacientes con diagnóstico previo de DM que tuvieron un promedio glicémico menor a 140 mg/dL. En la Figura 2 se observa la distribución de pacientes según diagnóstico relacionado a sus glicemias.

En los pacientes con hiperglicemia $>140$ $\mathrm{mg} / \mathrm{dL}$, aquellos con DM previa presentaron un promedio glicémico de 238,9 \pm 94,0 mg/dL, com-

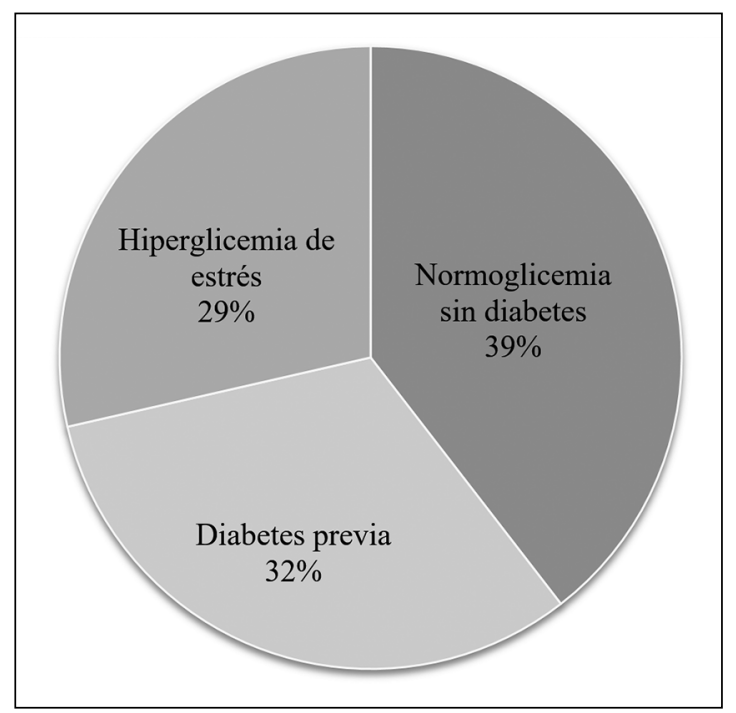

Figura 2. Diagnóstico metabólico en 210 pacientes adultos hospitalizados. Se muestra la distribución de 210 pacientes según diagnóstico metabólico. Cabe destacar que del total de los 67 pacientes con antecedente de diabetes previa, 14 de éstos $(20,9 \%)$ se encontraban en normoglicemia ( $<140 \mathrm{mg} / \mathrm{dL}$ ). 
Tabla 3. Número de pacientes con diabetes previa e hiperglicemia de estrés (HGE) en el grupo con glicemia mayor a $140 \mathrm{mg} / \mathrm{dL}$ según patología de ingreso

\begin{tabular}{|lccc|}
\hline Diagnóstico & Diabetes previa & HGE & p* \\
\hline Infeccioso & 13 & 17 & ns \\
\hline Oncohematológico & 4 & 18 & 0,03 \\
\hline Cardiovascular & 14 & 7 & 0,044 \\
\hline Neurológico no vascular & 6 & 4 & $\mathrm{~ns}$ \\
\hline Metabólico-Renal & 3 & 4 & $\mathrm{~ns}$ \\
\hline Quirúrgica & 10 & 9 & $\mathrm{~ns}$ \\
\hline Otros & 3 & 1 & $\mathrm{~ns}$ \\
\hline
\end{tabular}

*Se muestra significancia estadística de la diferencia según Chi cuadrado.

parado con $178,2 \pm 39,3 \mathrm{mg} / \mathrm{dL}$ en los pacientes con HGE (MD = 60,7 mg/dL, $\mathrm{p}=0,00001)$. Comparando los porcentajes de pacientes con DM previa y con HGE, la hiperglicemia $>180 \mathrm{mg} / \mathrm{dL}$ se encontró en 71,7 y $37,7 \%$, respectivamente, $(p=0,001)$. La hiperglicemia $>250 \mathrm{mg} / \mathrm{dL}$ se encontró en 40,0 \% de los pacientes con DM previa $y$ en $3,3 \%$ de aquellos con HGE ( $p=0,00001)$.

Al comparar el promedio de las glicemias y la distribución de las hiperglicemias según el diagnóstico principal, no se encontraron diferencias significativas entre los distintos grupos $(\mathrm{p}=0,221)$. Sin embargo, al realizar un análisis según la patología principal, los pacientes cardiovasculares con hiperglicemias $>140 \mathrm{mg} / \mathrm{dL}$ en un mayor número tenían DM previa (14 vs. 7 casos con HGE, $\mathrm{p}=0,044)$. En cambio, en los pacientes oncohematológicos se encontró una mayor proporción de casos con HGE que con $\mathrm{DM}$ previa (18 vs. 4 pacientes, $\mathrm{p}=0,003$ ). Esto se muestra en la Tabla 3.

Según el tipo de servicio de hospitalización, en los pacientes en unidades críticas el promedio glicémico fue de $175,4 \pm 85,9 \mathrm{mg} / \mathrm{dL}$, comparado con los pacientes en unidades básicas médico-quirúrgicas cuyo promedio fue de 155,0 $\pm 71,5 \mathrm{mg} / \mathrm{dL}$, con una MD de $20,3 \mathrm{mg} / \mathrm{dL}$ que no alcanza significancia estadística $(\mathrm{p}=0,086)$. Tampoco hubo diferencias al comparar las tasas de pacientes con hiperglicemia $>140 \mathrm{mg} / \mathrm{dL}$ entre los distintos servicios: 30 de 56 pacientes $(53,6 \%)$ en unidades críticas versus 83 de 154 (53,8\%) en unidades básicas $(\mathrm{p}=1)$. En cambio, efectivamente se encontraron diferencias significativas al evaluar la tasa de pacientes con hiperglicemia
$>180 \mathrm{mg} / \mathrm{dL}$ (que deben ser tratados según los consensos). Así, 24 de 56 pacientes (42,9\%) hospitalizados en unidades de críticos, comparado con 38 de 154 pacientes $(24,7 \%)$ en servicios de unidades básicas presentaron glicemias $>180$ $\mathrm{mg} / \mathrm{dL}(\mathrm{p}=0,016)$.

La duración de la estadía hospitalaria fue significativamente más larga en pacientes con hiperglicemia $>140 \mathrm{mg} / \mathrm{dL}$. Esta fue de 29,3 \pm 35,5 días para los pacientes con hiperglicemia y de $12,8 \pm 16$ días para los pacientes normoglicémicos (MD 16,5 días, $\mathrm{p}=0,00003$ ). Además, al evaluar exclusivamente a los pacientes hiperglicémicos, el tiempo de estadía fue de $20 \pm 29,1$ días para pacientes con DM previa versus $37,5 \pm 38,7$ días para aquellos con HGE (MD 17,5 días, $\mathrm{p}=0,008$ ). No se encontraron diferencias en el tiempo de estadía según diagnóstico $(\mathrm{p}=0,736)$, presencia de TFG $<60 \mathrm{mg} / \mathrm{dL}(\mathrm{p}=0,351)$ ni servicio donde ocurrió la hospitalización $(\mathrm{p}=0,346)$. Al introducir todas estas variables en un modelo de regresión lineal, la hiperglicemia $>140 \mathrm{mg} / \mathrm{dL}$ se mantuvo como la única variable asociada significativamente al tiempo de estadía hospitalaria $(\mathrm{p}=0,000036)$.

\section{Discusión}

El presente estudio muestra, en un corte transversal, la concentración de glucosa en sangre de pacientes adultos hospitalizados en distintos servicios de un hospital terciario y también el tipo de tratamiento que recibían para el control de la hiperglicemia. Concordante con la literatura internacional ${ }^{3}$, más de la mitad de los pacientes hos- 
pitalizados presenta cifras de glicemia superiores a $140 \mathrm{mg} / \mathrm{dL}$, correspondiendo aproximadamente la mitad de ellos a casos con DM previamente diagnosticada y la otra mitad a hiperglicemias de estrés ( 46,9 y $53,1 \%$, respectivamente).

Es llamativo que en este estudio, el 33,9\% de pacientes con hiperglicemia mayor a $180 \mathrm{mg} /$ dL se encontraba sin terapia alguna para control glicémico. Este hecho es preocupante, ya que esta situación se asocia a desenlaces adversos e incluso a mayor mortalidad y debieran ser tratados según normas de consensos internacionales ${ }^{1}$.

En el análisis según el diagnóstico de la patología principal, no se encontraron diferencias significativas en los promedios de hiperglicemia. Como dato interesante, y esperable por la fisiopatología de la enfermedad ateroesclerótica, la mayoría de los pacientes hiperglicémicos con diagnóstico cardiovascular corresponden a personas con DM previamente diagnosticada. En contraposición, en pacientes con diagnóstico oncohematológico, una significativa mayor proporción de pacientes con hiperglicemia corresponden a HGE. Este hecho era esperable, considerando la alta carga de enfermedad y el elevado uso de terapia esteroidal en este grupo de pacientes.

Otro hallazgo interesante de esta cohorte es la ausencia de diferencias en prevalencia de hiperglicemia entre unidades críticas y los servicios básicos. Sin embargo, al considerar la magnitud de la hiperglicemia, en realidad aparecen significativamente más hiperglicemias que deben ser tratadas en las unidades críticas. Esto también era esperable dado el mayor nivel de compromiso sistémico y gravedad de la enfermedad en los pacientes críticos.

El resultado más importante, concordante con la literatura internacional ${ }^{3,6}$ es la gran diferencia en estadía hospitalaria que se observa entre pacientes con normoglicemia, al compararlos con aquellos con hiperglicemia. Este tiempo se duplica, siendo más marcado en aquellos pacientes con hiperglicemia sin el diagnóstico previo de $\mathrm{DM}$, es decir se presentan con hiperglicemia del estrés (HGE). Esta observación podría estar asociada a múltiples factores, como la patología del diagnóstico principal, presencia de insuficiencia renal y estadía en cuidados críticos. Sin embargo, al analizar todas estas variables en un modelo de regresión lineal, ninguna resulta significativamente asociada al tiempo de estadía, a excepción de la hiperglicemia $>140 \mathrm{mg} / \mathrm{dL}$, que mantiene su relación con la prolongación del tiempo de estadía.

Una limitante del presente análisis es su carácter observacional de un estudio transversal sin un seguimiento de los pacientes, por lo que algunos de sus hallazgos podrían verse explicadas por factores no controlados. Sin embargo, los resultados tienen la lógica de los efectos nocivos de la hiperglicemia a nivel hospitalario y son concordantes con lo publicado previamente a nivel mundial.

A opinión de los autores, estos hallazgos ponen de manifiesto la muy alta prevalencia de hiperglicemia en un hospital. También destaca que muchos pacientes hacen una hiperglicemia del estrés que merece ser tratada en forma similar a quienes tienen el diagnóstico de DM previa. El reconocer esta condición es importante, porque la hiperglicemia aumenta el estrés oxidativo e inflamatorio y produce disfunción endotelial y del sistema inmune. Una hiperglicemia aumenta los desenlaces clínicos adversos y la mortalidad.

Es importante destacar la importancia del diagnóstico y la implementación de tratamientos adecuados para el control glicémico a nivel hospitalario. Es necesario implementar medidas educativas para todo el personal de salud con protocolos de manejo que deben ser evaluados periódicamente.

Agradecimientos: El equipo de investigadores quisiera agradecer a sus respectivas familias y seres queridos por el constante apoyo en su desarrollo profesional. Quisiéramos agradecer en particular a Carolina Torres, secretaria del Departamento de Nutrición, Diabetes y Metabolismo de la Pontificia Universidad Católica de Chile por su siempre necesario y gentil apoyo en todos los proyectos que se realizan en el Departamento. Agradecemos finalmente a todos quienes ayudaron en la recolección masiva de datos en el momento que se realizó el estudio: Margot Aliste, Mariana Boncompte, Álvaro Contreras, María Catalina Heusser, Javiera Perelli, Javier Vega.

\section{Referencias}

1. American Diabetes Association. Diabetes Care in the Hospital: Standards of Medical Care in Diabetes - 2020. Diabetes Care 2020; 43 (Supplement 1): S193-S202. 
2. Ministerio de Salud. Encuesta Nacional de Salud 20162017. Chile: MINSAL, 2017.

3. Umpierrez GE, Isaacs SD, Bazargan N, You X, Thaler LM, Kitabchi AE. Hyperglycemia: an independent marker of in-hospital mortality in patients with undiagnosed diabetes. J Clin Endocrinol Metab 2002; 87 (3): 978-82.

4. Saiepour D, Sehlin J, Oldenborg PA. Hyperglycemia-induced protein kinase $\mathrm{C}$ activation inhibits phagocytosis of C3b- and immunoglobulin g-opsonized yeast particles in normal human neutrophils. Exp Diabesity Res. 2003 Apr-Jun; 4 (2): 125-32.

5. Cosentino F, Hishikawa K, Katusic ZS, Lüscher TF. High glucose increases nitric oxide synthase expression and superoxide anion generation in human aortic endothelial cells. Circulation 1997; 96 (1): 25-8.

6. Gebreegziabher Y, McCullough P, Bubb C, Loney-Hutchinson L, Makaryus J, Anand N, et al. Admission Hyperglycemia and Length of Hospital Stay in Patients With Diabetes and Heart Failure: A Prospective Cohort Study. Congest Heart Fail 2008; 14 (3): 117-20.

7. Cheung NW, Li S, Ma G, Crampton R. The relationship between admission blood glucose levels and hospital mortality. Diabetologia 2008; 51 (6): 952-5.

8. Targher G, Dauriz M, Tavazzi L, Temporelli PL, Lucci D, Urso R. Prognostic Impact of In-Hospital Hyperglycemia in Hospitalized Patients With Acute Heart
Failure: Results of the IN-HF (Italian Network on Heart Failure) Outcome Registry. Int J Cardiol 2016; 203: 58793.

9. Van den Berghe G, Wouters P, Weekers F, Verwaest C, Bruyninckx F, Schetz M, et al. Intensive insulin therapy in critically ill patients. N Engl J Med 2001; 345 (19): 1359-67.

10. Van den Berghe G, Wilmer A, Hermans G, Meersseman W, Wouters PJ, Milants I, et al. Intensive insulin therapy in the medical ICU. N Engl J Med 2006; 354 (5): 449-61.

11. NICE-SUGAR Study Investigators, Finfer S, Chittock DR, Su SY, Blair D, Foster D, et al. Intensive versus conventional glucose control in critically ill patients. $\mathrm{N}$ Engl J Med 2009; 360 (13): 1283-97.

12. Turchin A, Matheny ME, Shubina M, Scanlon JV, Greenwood B, Pendergrass ML. Hypoglycemia and clinical outcomes in patients with diabetes hospitalized in the general ward. Diabetes Care 2009; 32 (7): 1153-7.

13. WHO Expert Committee on Physical Status: the Use and Interpretation of Anthropometry (1993: Ginebra, Suiza) \& World Health Organization. (1995). Physical status: the use of and interpretation of anthropometry, report of a WHO expert committee. World Health Organization.

14. Levey A, Stevens L, Schmid C, Zhang YL, Castro A, Feldman $\mathrm{H}$, et al. A new equation to estimate glomerular filtration rate. Ann Intern Med 2009; 150 (9): 604-12. 